\title{
The Multiplicative Zagreb Indices of Nanostructures and Chains
}

\author{
Wei Gao1, Mohammad Reza Farahani2, M. R. Rajesh Kanna ${ }^{3}$ \\ ${ }^{1}$ School of Information Science and Technology, Yunnan Normal University, Kunming, China \\ ${ }^{2}$ Department of Applied Mathematics, Iran University of Science and Technology, Tehran, Iran \\ ${ }^{3}$ Post Graduate Department of Mathematics, Maharani' Science College for Women, Mysore, India \\ Email: gaowei@ynnu.edu.cn,mrfarahani88@gmail.com,mr.rajeshkanna@gmail.com
}

Received 11 December 2015; accepted 5 April 2016; published 8 April 2016

Copyright (C) 2016 by authors and Scientific Research Publishing Inc.

This work is licensed under the Creative Commons Attribution International License (CC BY). http://creativecommons.org/licenses/by/4.0/

(c)

\begin{abstract}
In theoretical chemistry, the researchers use graph models to express the structure of molecular, and the Zagreb indices and multiplicative Zagreb indices defined on molecular graph $G$ are applied to measure the chemical characteristics of compounds and drugs. In this paper, we present the exact expressions of multiplicative Zagreb indices for certain important chemical structures like nanotube, nanostar and polyomino chain.
\end{abstract}

\section{Keywords}

Molecular Graph, The First Multiplicative Zagreb Index, The Second Multiplicative Zagreb Index, Nanotube, Nanostar, Polyomino Chain

\section{Introduction}

For the past 40 years, chemical graph theory, as an important branch of both computational chemistry and graph theory, has attracted much attention and the results obtained in this field have been applied in many chemical and pharmaceutical engineering applications. In these frameworks, the molecular is represented as a graph in which each atom is expressed as a vertex and covalent bounds between atoms are represented as edges between vertices. Topological indices were introduced to determine the chemical and pharmaceutical properties. Such indices can be regarded as score functions which map each molecular graph to a non-negative real number. There were many famous degree-based or distance-based indices such as Wiener index, PI index, Zagreb index, atom-bond connectivity index, Szeged index and eccentric connectivity index. Because of its wide engineering applications, many works contributed to determining the indices of special molecular graphs (see Yan et al., [1] and [2], Gao and Shi [3] and [4], Xi and Gao [5], Gao and Wang [6]-[8], Gao and Farahani [9], and Gao et al., [10] for more details). 
In our article, we only consider simple (molecular) graphs which are finite, loopless, and without multiple edges. Let $G=(V(G), E(G))$ be a graph in which the vertex set and edge set are expressed as $V(G)$ and $E(G)$, respectively. Here, each edge can be regarded as the subset of $V(G)$ with exactly two elements, and edge set $E(G)$ consists of all such edges. Readers can refer Bondy and Mutry [11] for any notations and terminologies used but not clearly explained in our paper.

The first Zagreb index could be regarded as one of the oldest graph invariants which was defined in 1972 by Gutman and Trinajsti [12] as

$$
M_{1}(G)=\sum_{v \in V(G)} d^{2}(v),
$$

where $d(v)$ is the degree vertex $v$ in $G$. Another alternative formulation for $M_{1}(G)$ is denoted as $\sum_{e=u v \in E(G)}(d(u)+d(v))$. And, the second Zagreb index was later introduced as

$$
M_{2}(G)=\sum_{e=u v \in E(G)}(d(u) d(v)) .
$$

As degree-based topological indices, the multiplicative version of these Zagreb indices of a graph $G$ is introduced by Gutman [13], and Ghorbani and Azimi [14] as:

$$
\begin{aligned}
& P M_{1}(G)=\prod_{e=u v \in E(G)}(d(u)+d(v)), \\
& P M_{2}(G)=\prod_{e=u v \in E(G)}(d(u) d(v)) .
\end{aligned}
$$

Here $P M_{1}(G)$ is the first multiplicative Zagreb index and $P M_{2}(G)$ is the second multiplicative Zagreb index. Several conclusions on these two classes of multiplicative Zagreb indices can be refered to Eliasi et al., [15], Xu et al., [16], and Farahani [17] and [18].

There have been many advances in Wiener index, Szeged index, PI index, and other degree-based or distancebased indices of molecular graphs, while the study of the first and second multiplicative Zagreb index of special chemical structures has been largely limited. Furthermore, nanotube, nanostar and polyomino chain are critical and widespread molecular structures which have been widely applied in medical science, chemical engineering and pharmaceutical fields. Also, these structures are the basic and primal structures of other more complicated chemical molecular structures. Based on these grounds, we have attracted tremendous academic and industrial interests in determining the multiplicative Zagreb indices of special family of nanotube, nanostar and polyomino chain from a computation point of view.

The contribution of our paper is three-folded. First, we focus on four classes of nanotubes: $V C_{5} C_{7}[p, q]$, $H_{5} C_{7}[p, q]$, polyhex zigzag $T U Z C_{6}$ and polyhex armchair $T U A C_{6}$, and the multiplicative Zagreb indices of these four classes of nanotubes are determined. Second, we compute the multiplicative Zagreb indices of dendrimer nanostar $D_{3}[n]$. At last, we calculate the multiplicative Zagreb indices of some special families of polyomino chains.

\section{Multiplicative Zagreb Indices of Nanotubes}

The purpose of this section is to yield the multiplicative Zagreb indices of certain special classes nanotubes. Our work in this part can be divided into two parts: 1) $V C_{5} C_{7}[p, q]$ and $H_{5} C_{7}[p, q]$ nanotubes; 2) zigzag $\mathrm{TUZC}_{6}$ and armchair $\mathrm{TUAC}_{6}$.

\subsection{Nanotubes Covered by $C_{5}$ and $C_{7}$}

In this subsection, we discuss $V C_{5} C_{7}[p, q]$ and $H C_{5} C_{7}[p, q]$ nanotubes which consisting of cycles $C_{5}$ and $C_{7}$ (or it is a trivalent decoration constructed by $C_{5}$ and $C_{7}$ in turn, and thus called $C_{5} C_{7}$-net). It can cover either a cylinder or a torus.

The parameter $p$ is denoted as the number of pentagons in the 1-st row of $V C_{5} C_{7}[p, q]$ and $H_{5} C_{7}[p, q]$. The vertices and edges in first four rows are repeated alternatively. In these nanotubes, and we set $q$ as the number of such repetitions. For arbitrary $p, q \in \mathbb{N}$, there exist $16 p$ edges and $6 p$ vertices in each period of 
$V C_{5} C_{7}[p, q]$ which are adjacent at the end of the molecular structure. By simple computation, we check that $\left|V\left(V C_{5} C_{7}[p, q]\right)\right|=16 p q+6 p$ and $\left|E\left(V C_{5} C_{7}[p, q]\right)\right|=24 p q+6 p$ since there are $6 p$ vertices with $d(v)=2$ and other $16 p q$ vertices with $d(v)=3$.

Furthermore, there are $8 p$ vertices and $12 p$ edges in any periods of $H_{5} C_{7}[p, q]$. We get $\left|V\left(H C_{5} C_{7}[p, q]\right)\right|=8 p q+5 p$ and $\left|E\left(H C_{5} C_{7}[p, q]\right)\right|=12 p q+5 p$ since there are $5 p$ vertices adjacent at the end of structure, and exists $q$ repetition and $5 p$ addition edges.

Let $\delta$ and $\Delta$ be the minimum and maximum degree of graph $G$, respectively. In the whole following context, for any graph $G$, its vertex set $V(G)$ and edge set $E(G)$ are divided into several partitions:

for any $i, 2 \delta(G) \leq i \leq 2 \Delta(G)$, let $E_{i}=\{e=u v \in E(G) \mid d(u)+d(v)=i\}$;

for any $j,(\delta)^{2} \leq j \leq(\Delta)^{2}$, let $E_{j}^{*}=\{e=u v \in E(G) \mid d(u) d(v)=j\}$;

for any $k, \delta \leq k \leq \Delta$, let $V_{k}=\{v \in V(G) \mid d(v)=k\}$.

Therefore, by omitting the single carbon atoms and the hydrogen, we infer two partitions

$V_{2}=\{v \in V(G) \mid d(v)=2\}$ and $V_{3}=\{v \in V(G) \mid d(v)=3\}$ for $V C_{5} C_{7}[p, q]$ and $H C_{5} C_{7}[p, q]$. Moreover, the edge set of $V C_{5} C_{7}[p, q]$ and $H C_{5} C_{7}[p, q]$ can be divided into the following three edge sets.

$E_{4}\left(\right.$ or $\left.E_{4}^{*}\right): d(u)=d(v)=2$;

$E_{6}\left(\right.$ or $\left.E_{9}^{*}\right): d(u)=d(v)=3$;

$E_{5}$ (or $\left.E_{6}^{*}\right), d(u)=2$ and $d(v)=3$.

Now, we state the main results in this subsection.

Theorem 1.

$$
\begin{gathered}
P M_{1}\left(V C_{5} C_{7}[p, q]\right)=6^{24 p q-6 p} 5^{12 p}, \\
P M_{2}\left(V C_{5} C_{7}[p, q]\right)=9^{24 p q-6 p} 6^{12 p}, \\
P M_{1}\left(H C_{5} C_{7}[p, q]\right)=6^{12 p q-4 p} 5^{8 p} 4^{p}, \\
P M_{2}\left(H C_{5} C_{7}[p, q]\right)=9^{12 p q-4 p} 6^{8 p} 4^{p} .
\end{gathered}
$$

Proof. First, considering nanotubes $V C_{5} C_{7}[p, q]$ for arbitrary $p, q \in N$. By analyzing its structure, we have $\left|V_{2}\right|=6 p,\left|V_{3}\right|=16 p q,\left|E_{5}\right|=\left|E_{6}^{*}\right|=12 p$ and $\left|E_{6}\right|=\left|E_{9}^{*}\right|=24 p q-6 p$. In terms of the definitions of multiplicative version of these Zagreb indices, we infer

$$
\begin{gathered}
P M_{1}\left(V C_{5} C_{7}[p, q]\right)=\prod_{e=u v \in E_{6}}(d(u)+d(v)) \times \prod_{e=u v \in E_{5}}(d(u)+d(v))=6^{\left|E_{6}\right|} 5^{\left|E_{5}\right|} . \\
P M_{2}\left(V C_{5} C_{7}[p, q]\right)=\prod_{e=u v \in E_{6}}(d(u) d(v)) \times \prod_{e=u v \in E_{5}}(d(u)+d(v))=9^{\left|E_{6}\right|} 6^{\left|E_{5}\right|} .
\end{gathered}
$$

Second, we consider nanotube $\mathrm{HC}_{5} C_{7}[p, q]$ for arbitrary $p, q \in \mathbb{N}$. According to its chemical structure, we verify $\left|V_{2}\right|=5 p,\left|V_{3}\right|=8 p q,\left|E_{4}\right|=\left|E_{4}^{*}\right|=p,\left|E_{5}\right|=\left|E_{6}^{*}\right|=8 p$, and $\left|E_{6}\right|=\left|E_{9}^{*}\right|=12 p q-4 p$. Therefore, by means of the definitions of multiplicative version of these Zagreb indices, we get

$$
\begin{aligned}
& P M_{1}\left(H C_{5} C_{7}[p, q]\right) \\
& =\prod_{e=u v \in E_{6}}(d(u)+d(v)) \times \prod_{e=u v \in E_{5}}(d(u)+d(v)) \times \prod_{e=u v \in E_{4}}(d(u)+d(v)) \\
& =6^{E_{6} \mid} 5^{E_{5} \mid} 4^{E_{4} \mid} . \\
& P M_{2}\left(H C_{5} C_{7}[p, q]\right) \\
& =\prod_{e=u v E_{6}}(d(u) d(v)) \times \prod_{e=u v \in E_{5}}(d(u)+d(v)) \times \prod_{e=u v \in E_{4}}(d(u)+d(v)) \\
& =9^{\left|E_{6}\right|} 5^{E_{5} \mid} 4^{\left|E_{4}\right|} .
\end{aligned}
$$

\subsection{Two Classes of Polyhex Nanotubes}

We study the multiplicative version of polyhex nanotubes: zigzag $T^{2} U Z C_{6}$ and armchair $T_{U A C}$ in this sub- 
section. We use parameter $m \in \mathbb{N}$ to denote the number of hexagons in the 1-st row of the $T U Z C_{6}$ and $T_{U A C}$. Analogously, the positive integer $n$ is used to express the number of hexagons in the 1-st column of the 2D-lattice of $T U Z C_{6}$ and $T U A C_{6}$. In view of structure analysis, we conclude

$\left|V\left(T U Z C_{6}\right)\right|=\left|V\left(T U A C_{6}\right)\right|=2 m(n+1)$ and $\left|E\left(T U Z C_{6}\right)\right|=\left|E\left(T U A C_{6}\right)\right|=3 m n+2 m$.

Clearly, the degree of vertex in polyhex nanotubes can't exceed three. For nanotubes $\operatorname{TUZC}_{6}[m, n]$ with any $m, n \in \mathbb{N}$, we infer $\left|V_{2}\right|=2 m,\left|V_{3}\right|=2 m n,\left|E_{5}\right|=\left|E_{6}^{*}\right|=4 m$ and $\left|E_{6}\right|=\left|E_{9}^{*}\right|=3 m n-2 m$. Moreover, for nanotube $T_{U A C}[m, n]$ with any $m, n \in \mathbb{N}$, we get $\left|V_{2}\right|=2 m,\left|V_{3}\right|=2 m n,\left|E_{4}\right|=\left|E_{4}^{*}\right|=m,\left|E_{5}\right|=\left|E_{6}^{*}\right|=2 m$ and $\left|E_{6}\right|=\left|E_{9}^{*}\right|=3 m n-m$. Therefore, the results stated as follows are obtained by means of above discussions and the definitions of multiplicative Zagreb indices.

\section{Theorem 2.}

$$
\begin{gathered}
P M_{1}\left(\text { TUZC }_{6}[m, n]\right)=6^{3 m n-2 m} 5^{4 m}, \\
P M_{2}\left(\text { TUZC }_{6}[m, n]\right)=9^{3 m n-2 m} 6^{4 m}, \\
P M_{1}\left(\text { TUAC }_{6}[m, n]\right)=6^{3 m n-m} 5^{2 m} 4^{m}, \\
P M_{2}\left(\text { TUAC }_{6}[m, n]\right)=9^{3 m n-m} 6^{2 m} 4^{m} .
\end{gathered}
$$

\section{Multiplicative Zagreb Indices of Dendrimer Nanostars}

Dendrimer is a basic structure in nanomaterials. In this section, for any $n \in \mathbb{N}, D_{3}[n]$ is denoted as the $n$-th growth of dendrimer nanostar. We aim to determine multiplicative Zagreb indices of dendrimer nanostar $D_{3}[n]$ (its structure can be referred to Figure 1 for more details).

This class of dendrimer nanostar has a core presented in Figure 1 and we call an element as a leaf. It is not difficult to check that a leaf is actually consisted of $C_{6}$ or chemically benzene, and $D_{3}[n]$ is constituted by adding $3 \cdot 2^{n}$ leafs in the $n$-th growth of $D_{3}[n-1]$. Therefore, there are in all $3 \cdot 2^{n+1}-3$ leafs $\left(C_{6}\right)$ in the dendrimer $D_{3}[n]$. The main contribution in this section can be stated as follows.

Theorem 3.

$$
\begin{gathered}
P M_{1}\left(D_{3}[n]\right)=4^{15 \cdot 2^{n}-6} 5^{12\left(2^{n+1}-1\right)} 6^{9 \cdot 2^{n}-6}, \\
P M_{2}\left(D_{3}[n]\right)=3^{3 \cdot 2^{n}} 4^{6\left(2^{n+1}-1\right)} 6^{12\left(2^{n+1}-1\right)} 9^{9 \cdot 2^{n}-6} .
\end{gathered}
$$

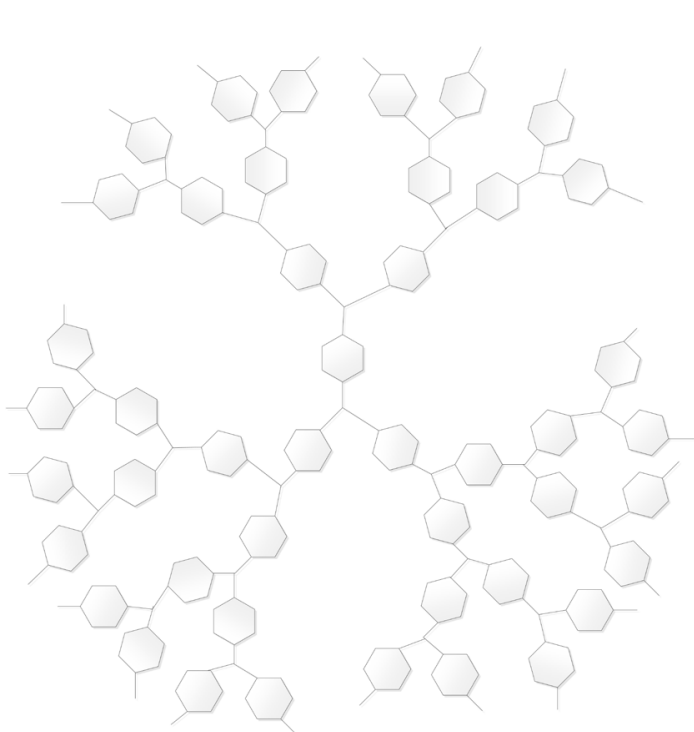

Figure 1. The structure of 2-dimensional of dendrimer nanostar $D_{3}[n]$. 
Proof. Let $V_{i}[n]$ be the number of vertices with degree $i(i \in\{1,2,3,4\})$ in $D_{3}[n]$. In terms of hierarchy structural of $D_{3}[n]$, we deduce $V_{1}[n+1]=2 V_{1}[n]=3 \cdot 2^{n+1}, V_{2}[n+1]=V_{2}[n]+12 \cdot 2^{n+1}$ and $V_{3}[n+1]=V_{3}[n]+6 \cdot 2^{n+1}+V_{1}[n]$. Hence, by means of the induction on $n$ with $V_{1}[0]=3, V_{2}[0]=12$ and $V_{3}[0]=7$, we get $V_{2}[n+1]=12\left(2^{n+2}-1\right)$ and $V_{3}[n+1]=15 \cdot 2^{n+1}-8$.

Set $E_{3}^{*}=\left\{e=u v \in E\left(D_{3}[n]\right) \mid d(u)=3, d(v)=1\right\}$. We infer

$$
\begin{gathered}
\left|E_{3}^{*}\right|=3 \cdot 2^{n}, \\
\left|E_{4}^{*}\right|=\left|E_{4}\right|=6\left(2^{n+1}-1\right), \\
\left|E_{6}^{*}\right|=\left|E_{5}\right|=12\left(2^{n+1}-1\right), \\
\left|E_{9}^{*}\right|=\left|E_{6}\right|=9 \cdot 2^{n}-6, \\
\left|E_{4}\right|=\left|E_{3}^{*}\right|+\left|E_{4}^{*}\right|=15 \cdot 2^{n}-6 .
\end{gathered}
$$

Therefore, the expected results are obtained by the definition of the first and the second multiplicative Zagreb index.

\section{Multiplicative Zagreb Indices of Polyomino Chains}

From the perspective of mathematical, a polyomino system can be considered as a finite 2-connected plane graph in which each interior cell is surrounded by a $C_{4}$. In other words, it can be regarded as an edge-connected union of cells in the planar square lattice. For instance, polyomino chain is a special polyomino system in which the joining of the centers (denoted $c_{i}$ as the center of the $i$-th square) of its adjacent regular forms a path $C_{1} C_{2} \cdots c_{n}$. Let $\mathcal{P}_{n}$ be the set of polyomino chains with $n$ squares. We have $\left|E\left(P C_{n}\right)\right|=3 n+1$ for each $P C_{n} \in \mathcal{P}_{n} . P C_{n}$ is called a linear chain expressed as $L C_{n}$ if the subgraph of $P C_{n}$ induced by $V_{3}$ has exactly $n-2$ squares. Moreover, $P C_{n}$ is called a zig-zag chain denoted as $Z C_{n}$ if the subgraph of $P C_{n}$ induced by $V_{>2}$ (all the vertices with degree larger than two) is a path has exactly $n-1$ edges.

The branched or angularly connected squares in a polyomino chain are called a kink, and a maximal linear chain in a polyomino chain including the kinks and terminal squares at its end is called a segment represented by $S$. We use $l(S)$ to denote the length of $S$ which is determined by the number of squares in $S$. Assume a polyomino chain consists of a sequence of segments $S_{1}, S_{2}, \cdots, S_{m}$ with $m \geq 1$, and we denote $l\left(S_{i}\right)=l_{i}$ for $i \in\{1,2, \cdots, m\}$ with property that $\sum_{i=1}^{m} l_{i}=n+m-1$. For arbitrary segment $S$ in a polyomino chain, we have $2 \leq l(S) \leq n$. Specially, we get $m=1$ and $l_{1}=n$ for a linear chain $L C_{n}$, and $m=n-1$ and $l_{i}=2$ for a zig-zag chain $Z C_{n}$.

The theorems presented in the below reveal clearly how the multiplicative Zagreb indices of certain families of polyomino chain are expressed.

Theorem 4. Let $L C_{n}, Z C_{n}$ be the polyomino chains presented above. Then, we get

$$
\begin{gathered}
P M_{1}\left(L C_{n}\right)= \begin{cases}256, & n=1 \\
10000 \cdot 6^{3 n-5}, & n \geq 2 .\end{cases} \\
P M_{2}\left(L C_{n}\right)= \begin{cases}256, & n=1 \\
20736 \cdot 9^{3 n-5}, & n \geq 2 .\end{cases} \\
P M_{1}\left(Z C_{n}\right)= \begin{cases}256, & n=1 \\
10000 \cdot 6^{2(m-1)} 7^{2} 8^{m-2}, & n \geq 2 .\end{cases} \\
P M_{2}\left(Z C_{n}\right)= \begin{cases}256, & n=1 \\
20736 \cdot 9^{2(m-1)} 12^{2} 16^{m-2}, & n \geq 2 .\end{cases}
\end{gathered}
$$

Proof. The results are obvious for $n=1$, and we only focus on $n \geq 2$ in the following discussion. It is not hard to check that $\left|E\left(L C_{n}\right)\right|=\left|E\left(Z C_{n}\right)\right|=3 n+1$.

For the polyomino chain $L C_{n}$, we obtain $\left|E_{4}\right|=\left|E_{4}^{*}\right|=2,\left|E_{5}\right|=\left|E_{6}^{*}\right|=4$ and $\left|E_{6}\right|=\left|E_{9}^{*}\right|=3 n-5$. By the 
definitions of multiplicative Zagreb indices, we have

$$
\begin{aligned}
& P M_{1}\left(L C_{n}\right)=4^{2} 5^{4} 6^{3 n-5}, \\
& P M_{2}\left(L C_{n}\right)=4^{2} 6^{4} 9^{3 n-5} .
\end{aligned}
$$

By the same fashion, we yield

$$
\begin{gathered}
P M_{1}\left(Z C_{n}\right)=4^{2} 5^{4} 6^{2(m-1)} 7^{2} 8^{3 n-2 m-5}, \\
P M_{2}\left(Z C_{n}\right)=4^{2} 6^{4} 9^{2(m-1)} 12^{2} 16^{3 n-2 m-5} .
\end{gathered}
$$

The expected results are got from the fact $m=n-1$ for $Z C_{n}$.

Theorem 5. Let $P C_{n}^{1} \quad(n \geq 3)$ be a polyomino chain with $n$ squares and two segments which $l_{1}=2$ and $l_{2}=n-1$. Then, we have

$$
\begin{gathered}
P M_{1}\left(P C_{n}^{1}\right)= \begin{cases}88200000, & n=3 \\
17150000 \cdot 6^{3 n-9}, & n \geq 4 .\end{cases} \\
P M_{2}\left(P C_{n}^{1}\right)= \begin{cases}241864704, & n=3 \\
1719926784 \cdot 9^{3 n-10}, & n \geq 4 .\end{cases}
\end{gathered}
$$

Proof. For $n=3$, it is trivial. For $n \geq 4$, we obtain $\left|E_{4}\right|=\left|E_{4}^{*}\right|=2,\left|E_{5}\right|=\left|E_{6}^{*}\right|=5, \quad\left|E_{8}^{*}\right|=1, \quad\left|E_{7}\right|=\left|E_{12}^{*}\right|=3$, $\left|E_{9}^{*}\right|=3 n-10$ and $\left|E_{6}\right|=\left|E_{9}^{*}\right|+\left|E_{8}^{*}\right|=3 n-9$. Therefore, by means of simply calculation, we obtain the desired results.

Theorem 6. Let $P C_{n}^{2}$ be a polyomino chain with $n$ squares and $m$ segments $S_{1}, S_{2}, \cdots, S_{m} \quad(m \geq 3)$ such that $l_{1}=l_{m}=2$ and $l_{2}, \cdots, l_{m-1} \geq 3$. Then

$$
\begin{gathered}
P M_{1}\left(P C_{n}^{2}\right)=16 \cdot 5^{2 m} 6^{3 n-6 m+5} 7^{4 m-6}, \\
P M_{2}\left(P C_{n}^{2}\right)=1296 \cdot 6^{2 m} 12^{4 m-6} 9^{3 n-6 m+3} .
\end{gathered}
$$

Proof. For this chemical structure, we get $\left|E_{4}\right|=\left|E_{4}^{*}\right|=2,\left|E_{5}\right|=\left|E_{6}^{*}\right|=2 m,\left|E_{8}^{*}\right|=2,\left|E_{7}\right|=\left|E_{12}^{*}\right|=4 m-6$, $\left|E_{9}^{*}\right|=3 n-6 m+3$ and $\left|E_{6}\right|=\left|E_{9}^{*}\right|+\left|E_{8}^{*}\right|=3 n-6 m+5$. Therefore, in view of the definitions of multiplicative Zagreb indices, we obtain the desired results.

The last two results obtained using similarly tricks.

Corollary 1. Let $P C_{n}^{3} \quad(n \geq 4)$ be a polyomino chain with $n$ squares and $m$ segments $S_{1}, S_{2}, \cdots, S_{m} \quad(m \geq 3)$ such that $l_{1}=2, l_{2}, \cdots, l_{m} \geq 3$ or $l_{m}=2, l_{1}, l_{2}, \cdots, l_{m-1} \geq 3$. Then

$$
\begin{aligned}
& P M_{1}\left(P C_{n}^{3}\right)=16 \cdot 5^{2 m+1} 6^{3 n-6 m+4} 7^{4 m-5}, \\
& P M_{2}\left(P C_{n}^{3}\right)=16 \cdot 6^{2 m+1} 9^{3 n-6 m+4} 12^{4 m-5} .
\end{aligned}
$$

Corollary 2. Let $P C_{n}^{4}$ be a polyomino chain with $n$ squares and $m$ segments $S_{1}, S_{2}, \cdots, S_{m} \quad(m \geq 3)$ such that $l_{i} \geq 3 \quad(i=\{1, \cdots, m\})$. Then

$$
\begin{aligned}
& P M_{1}\left(P C_{n}^{4}\right)=16 \cdot 5^{2 m+2} 6^{3 n-6 m+1} 7^{4 m-4}, \\
& P M_{2}\left(P C_{n}^{4}\right)=16 \cdot 6^{2 m+2} 9^{3 n-6 m+1} 12^{4 m-4} .
\end{aligned}
$$

\section{Conclusions and Further Work}

The purpose of this paper is to discuss the multiplicative Zagreb indices of several chemical structures, and these molecular graphs we consider here are fundamentally and commonly used in chemical engineering. Specifically, the contributions in this report can be concluded into three aspects: first, we compute the multiplicative Zagreb indices of four classes of nanotubes; then, the multiplicative Zagreb indices of dendrimer nanostars $D_{3}[n]$ are calculated; at last, we also discuss some families of polyomino chains. As multiplicative Zagreb indices can been used in QSPR/QSAR study and play a crucial role in analyzing both the boiling point and melting point for medicinal drugs and chemical compounds, the results obtained in our paper illustrate the 
promising prospects of application for medical, pharmacal, biological and chemical sciences.

A closely related concept of the Zagreb index is the Estrada index (see Shang [19] and [20] for more details) and the techniques used in our paper can be potentially applicable to the Estrada indices. The Estrada index of special chemical graph structures can be considered in the further works.

\section{Acknowledgements}

We thank all the reviewers for their constructive comments in improving the quality of this paper. Research is supported partially by NSFC (No. 11401519).

\section{References}

[1] Yan, L., Li, Y., Gao, W. and Li, J.S. (2014) On the Extremal Hyper-Wiener Index of Graphs. Journal of Chemical and Pharmaceutical Research, 6, 477-481.

[2] Yan, L., Li, J.S. and Gao, W. (2014) Vertex PI Index and Szeged Index of Certain Special Molecular Graphs. The Open Biotechnology Journal, 8, 19-22. http://dx.doi.org/10.2174/1874070701408010019

[3] Gao, W. and Shi, L. (2014) Wiener Index of Gear fan Graph and Gear Wheel Graph. Asian Journal of Chemistry, 26, 3397-3400.

[4] Gao, W. and Shi, L. (2015) Szeged Related Indices of Unilateral Polyomino Chain and Unilateral Hexagonal Chain. IAENG International Journal of Applied Mathematics, 45, 138-150.

[5] Xi, W.F. and Gao, W. (2014) Geometric-Arithmetic Index and Zagreb Indices of Certain Special Molecular Graphs. Journal of Advances in Chemistry, 10, 2254-2261.

[6] Gao, W. and Wang, W.F. (2014) Second Atom-Bond Connectivity Index of Special Chemical Molecular Structures. Journal of Chemistry, 2014, Article ID: 906254. http://dx.doi.org/10.1155/2014/906254

[7] Gao, W. and Wang, W.F. (2015) The Vertex Version of Weighted Wiener Number for Bicyclic Molecular Structures. Computational and Mathematical Methods in Medicine, 2015, Article ID: 418106. http://dx.doi.org/10.1155/2015/418106

[8] Gao, W. and Wang, W.F. (2014) Revised Szeged Index and Revised Edge-Szeged Index of Special Chemical Molecular Structures. Journal of Interdisciplinary Mathematics, 4, 417-425.

[9] Gao, W. and Farahani, M.R. (2016) Degree-Based Indices Computation for Special Chemical Molecular Structures Using Edge Dividing Method. Applied Mathematics and Nonlinear Sciences, 1, 94-117.

[10] Gao, Y., Gao, W. and Liang, L. (2014) Revised Szeged Index and Revised Edge Szeged Index of Certain Special Molecular Graphs. International Journal of Applied Physics and Mathematics, 4, 417-425. http://dx.doi.org/10.17706/ijapm.2014.4.6.417-425

[11] Bondy, J.A. and Mutry, U.S.R. (2008) Graph Theory. Spring, Berlin. http://dx.doi.org/10.1007/978-1-84628-970-5

[12] Gutman, I. and Trinajsti, N. (1972) Graph Theory and Molecular Orbitals. III. Total $\varphi$-Electron Energy of Alternant Hydrocarbons. Chemical Physics Letters, 17, 535-538. http://dx.doi.org/10.1016/0009-2614(72)85099-1

[13] Gutman, I. (2011) Multiplicative Zagreb Indices of Trees. Bulletin of the International Mathematical Virtual Institute, 1, 13-19.

[14] Ghorbani, M. and Azimi, N. (2012) Note on Multiple Zagreb Indices. Iranian Journal of Mathematical Chemistry, 3, 137-143.

[15] Eliasi, M., Iranmanesh, A. and Gutman, I. (2012) Multiplicative Versions of First Zagreb Index. MATCH Communications in Mathematical and in Computer Chemistry, 68, 217-230.

[16] Xu, K. and Das, K.Ch. (2012) Trees, Unicyclic, and Bicyclic Graphs Extremal with Respect to Multiplicative Sum Zagreb Index. MATCH Communications in Mathematical and in Computer Chemistry, 68, 257-272.

[17] Farahani, M.R. (2014) On Multiple Zagreb Indices of Circumcoronene Homologous Series of Benzenoid. Chemical Physics Research Journal, 7, 277-281.

[18] Farahani, M.R. (2015) Multiplicative Versions of Zagreb Indices of $\mathrm{TUSC}_{4} C_{8}(S)$. Journal of Chemistry and Materials Research, 2, 67-70.

[19] Shang, Y.L. (2015) Laplacian Estrada and Normalized Laplacian Estrada Indices of Evolving Graphs. PLoS ONE, 10, E0123426. http://dx.doi.org/10.1371/journal.pone.0123426

[20] Shang, Y.L. (2015) The Estrada Index of Evolving Graphs. Applied Mathematics and Computation, 250, 415-423. http://dx.doi.org/10.1016/j.amc.2014.10.129 